\title{
Outburst activity in comets
}

\section{Continuous monitoring of comet 29P/Schwassmann-Wachmann 1}

\author{
J. M. Trigo-Rodríguez ${ }^{1,2}$, E. García-Melendo ${ }^{3}$, B. J. R. Davidsson ${ }^{4}$, A. Sánchez ${ }^{5}$, D. Rodríguez ${ }^{6}$, \\ J. Lacruz ${ }^{7}$, J. A. De los Reyes ${ }^{8}$, and S. Pastor ${ }^{8}$ \\ ${ }^{1}$ Institute of Space Sciences (CSIC), Campus UAB, Facultat de Ciències, Torre C-5, parells, 2a planta, 08193 Bellaterra (Barcelona), \\ Spain \\ 2 Institut d'Estudis Espacials de Catalunya (IEEC), Ed. Nexus, Gran Capità 2-4, 08034 Barcelona, Spain \\ e-mail: trigo@ieec.uab.es \\ Esteve Duran Observatory Foundation, Montseny 42, 08553 Seva, Barcelona, Spain \\ 4 Department of Physics and Astronomy, Uppsala University, Box 515, 75120, Uppsala, Sweden \\ 5 Gualba Astronomical Observatory (MPC442), Barcelona, Spain \\ ${ }^{6}$ Guadarrama Observatory (MPC458), Madrid, Spain \\ 7 La Cañada Observatory (MPC J87), Ávila, Spain \\ ${ }^{8}$ Observatorio Astronómico Municipal de Murcia (J76), La Murta, Spain
}

Received 13 September 2007 / Accepted 19 March 2008

\section{ABSTRACT}

\begin{abstract}
Aims. We carried out a continuous monitoring of comet 29P/Schwassmann-Wachmann 1 by using medium aperture telescopes with the aim of studying the activity and outburst mechanisms of this comet on the basis of photometric variations.

Methods. We used a standardized method to obtain the coma photometry in the $R$ filter of the Johnson-Kron-Cousins system. Some abrupt changes observed in the brightness of SW1 suggest important variations in surface activity with time.

Results. During our 2002-2007 observational campaign we detected 28 outbursts (of 1 mag or larger) in 29P/SchwassmannWachmann 1. A typical outburst is characterized by a rapid increase towards maximum (in a few hours) and a slower decrease toward the quiescent level (in 3-4 days). Given the effective observing time, the average outburst rate is 7.3 events per year. Despite well-sampled data, no signs of a clear periodicity in the outburst occurrence has been found, thus confirming the unpredictability of the activity of this comet.
\end{abstract}

Key words. comets: individual: 29P/Schwassmann-Wachmann 1 - Kuiper Belt - meteors, meteoroids

\section{Introduction}

Comet and Centaur object 29P/Schwassmann-Wachmann 1 (hereafter SW1) has exhibited abrupt increases in its brightness (so-called outbursts) ever since its discovery in 1925. In fact, SW1 is usually considered the archetype of comets exhibiting unusual changes in their coma appearance and brightness. During an outburst, its coma brightness typically increases by 2 to 5 mag. Similar outbursts have been observed in other periodic comets, but are typically associated with splitting (Sekanina 1982; Sekanina et al. 1996; Green 2000). In this paper we performed a continuous coverage of SW1 activity during 2002-2007 to study the amplitude and possible frequency of these fascinating events. Recently, an extraordinary outburst in comet 17P/Holmes has been reported (Green et al. 2007; Trigo-Rodríguez et al. 2007, 2008), which our group has studied thoroughly and which will be discussed in a future paper.

SW1 moves along a quasi-circular orbit with eccentricity $e \sim 0.044$ and semimajor axis $a \sim 6$ AU (Marsden $\&$ Williams 2003). Being so far away from the Sun, its surface is below the sublimation temperature of water ice, and other physical processes should play a role for generating outbursts. Froeschlé et al. (1983) first suggested that the continuous changes in its light curve could be associated with crystallization of amorphous ice. Years later his hypothesis was strengthened by the detection of $\mathrm{CO}$ outgassing that was naturally explained by the release of volatiles trapped in the amorphous ice (Senay \& Jewitt 1994). The important role of crystallization of ice in cometary outbursts has been modeled by Prialnik (2002), while the importance of $\mathrm{CO}$ as a driver of cometary activity at this solar distance was pointed out by Prialnik \& Bar-Nun (1990) and discussed further by Gunnarsson (2003). We were encouraged by these previous works in order to perform a continuous coverage of the SW1 activity to learn more about the processes that take place in the coma after an outburst. Because of the energy released during explosive activity it is possible that $\mu \mathrm{m}$-sized grains are able to leave the surface, forming a typical fan that is detectable even by using medium-sized telescopes (Fig. 1). The brightness of the coma during the massive release of dust increases significantly. In fact, during our observations we detected outbursts with increases of 2-4 mag in brightness, but up to 8 mag outbursts have already been reported (Sekanina 1982; Wyckoff 1982). In the present paper, we describe 28 outbursts detected as a result of a 6-year monitoring of this comet. In Sect. 2 the observational data and reduction procedures are compiled. Section 3 shows the results obtained. In Sect. 4 we discuss the implications of our observations for explaining cometary activity and dust processes that take place in the coma. 

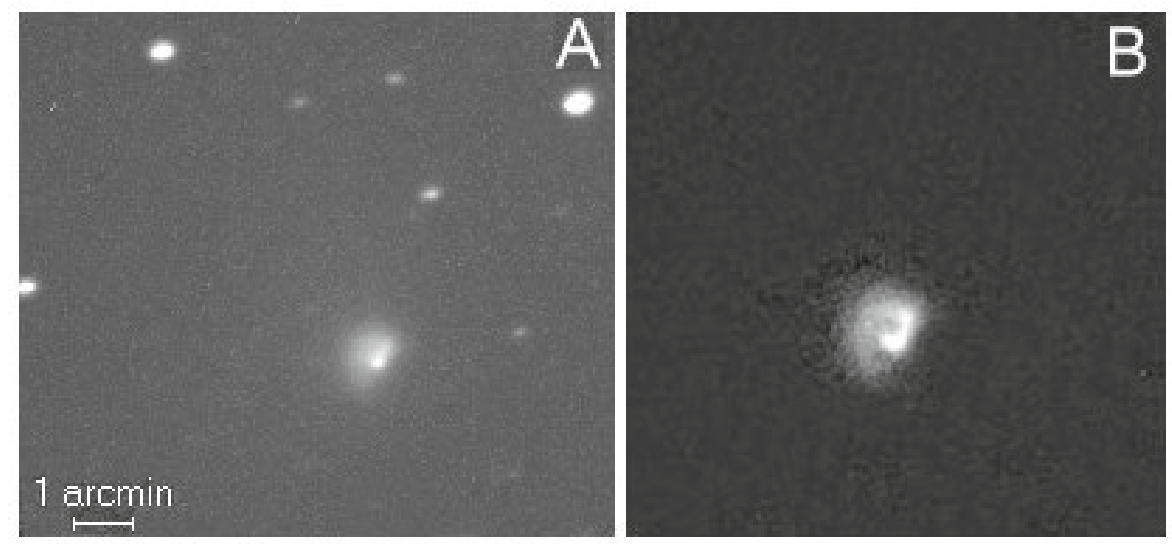

Fig. 1. Impressive jet detected 2003 August 30.9 recorded from Gualba Observatory (MPC 442). a) Original image. b) The jet enhanced on the same scale by using a Larson-Sekanina filter. Image resolution is $0.9 \mathrm{arcsec} / \mathrm{pixel}$.

Table 1. Observatories and people involved in our SW1 coverage.

\begin{tabular}{|c|c|c|c|c|c|}
\hline Observatory & MPC code & Observer & Telescope (s) & Long $\left({ }^{\circ}\right)$ & Lat $\left(^{\circ}\right)$ \\
\hline Gualba, Barcelona & 442 & A. Sánchez & $\begin{array}{l}\text { SC } 30.5 \mathrm{f} / 4 \\
\& \text { SC } 36.0 \mathrm{f} / 6\end{array}$ & $2^{\circ} 31^{\prime} 15^{\prime \prime} \mathrm{E}$ & $41^{\circ} 43^{\prime} 16^{\prime \prime} \mathrm{N}$ \\
\hline Guadarrama, Madrid & 458 & D. Rodríguez & $\mathrm{SC} 20 \mathrm{f} / 10$ & $4^{\circ} 01^{\prime} 10^{\prime \prime} \mathrm{W}$ & $40^{\circ} 30^{\prime} 50^{\prime \prime} \mathrm{N}$ \\
\hline $\begin{array}{l}\text { Fundació Observatori Esteve } \\
\text { Duran (FOED), Barcelona }\end{array}$ & - & E. García-Melendo & C $61 \mathrm{f} / 6$ & $2^{\circ} 15^{\prime} 50^{\prime \prime} \mathrm{E}$ & $41^{\circ} 48^{\prime} 32^{\prime \prime} \mathrm{N}$ \\
\hline La Murta Observatory, Murcia & $\mathrm{J} 76$ & $\begin{array}{l}\text { José A. de los Reyes } \\
\text { Sensi Pastor }\end{array}$ & $\mathrm{SC} 41 \mathrm{f} / 10$ & $1^{\circ} 12^{\prime} 10^{\prime \prime} \mathrm{W}$ & $37^{\circ} 50^{\prime} 24^{\prime \prime} \mathrm{N}$ \\
\hline La Cañada Observatory & J87 & J. de la Cruz & SC $20 \mathrm{f} / 10$ & $4^{\circ} 29^{\prime} 30^{\prime \prime} \mathrm{W}$ & $40^{\circ} 36^{\prime} 18^{\prime \prime} \mathrm{N}$ \\
\hline $\begin{array}{l}\text { Montsec Astronomical } \\
\text { Observatory (OAdM), Lleida }\end{array}$ & - & $\begin{array}{l}\text { D. Fernández } \\
\text { J. M. Trigo-Rodríguez }\end{array}$ & SC 80.0 f/10 & $0^{\circ} 43^{\prime} 46^{\prime \prime} \mathrm{E}$ & $42^{\circ} 03^{\prime} 05^{\prime \prime} \mathrm{N}$ \\
\hline $\begin{array}{l}\text { Montseny Astronomical } \\
\text { Observatory, Girona }\end{array}$ & B06 & J. M. Trigo-Rodríguez & N 18 f/6 & $2^{\circ} 31^{\prime} 14^{\prime \prime} \mathrm{E}$ & $41^{\circ} 43^{\prime} 17^{\prime \prime} \mathrm{N}$ \\
\hline
\end{tabular}

\section{Observational data and reduction procedure}

To follow up the outburst activity of comet SW1, we used CCD photometry obtained by several amateur and professional observatories (Table 1). We followed the same reduction procedure as described previously by Kidger (2002) using a 10 arcsec aperture and reducing data against USNO A2.0 "R" stars. Although this is not a photometric catalog, its accuracy is good enough (about $0.2 \mathrm{mag}$ ) for follow up of cometary activity, as pointed out by Kidger (2003). In fact, the typical standard deviation among data points obtained from different telescopes is between 0.05 and $0.1 \mathrm{mag}$. The software typically used for 10 arcsec photometric reduction was Astrometrica (Raab 2005). The results were systematically tested by using an additional software package called LAIA (Laboratory for Astronomical Image Analysis) successfully tested for obtaining high-precision stellar photometry (García-Melendo \& Clement 1997; Escolà-Sirisi et al. 2005). The corresponding spatial scale was typically between 0.8 to 1.9 arcsec/pixel, which corresponds respectively to a resolution of about 33400 and $79500 \mathrm{~km} /$ pixel at the Earth-comet distance.

A total of 754 photometric measurements of SW1 have been considered in the present paper, although observations are still continuing. The data were obtained by eight experienced observers on 365 nights extending between June 2002 and December 2007.

\section{Results}

All photometric observations considered in the present work are shown in Fig. 6. This figure also shows five close-up windows on selected outbursts. Measurements were only severely interrupted by the SW1 conjunctions with the Sun occurring during the observing period. Below we describe some of the most important outbursts that were detected and monitored during the time interval.

In August 2003 on JD 2452876.5 the comet exhibited a bright outburst that was covered very well by our team. Two observations separated by three days show an increase from magnitude +15.2 to +13.0 in the 10 arcsec standard photometry field that we use. After that, the decay was gradual and the magnitude decreased to +14.9 in the following four days, practically reaching the pre-outburst level. Looking at Fig. 6 there is apparently a variation in the rate of change during magnitude decrease for different outbursts. These will be analyzed in detail in a forthcoming paper.

The brightest outburst noticed during our 2002-2007 survey was recorded on September 17, 2004 on JD 2453266.4. During three weeks of monitoring, the comet was really faint (mag +15.6), very diffuse, and did not have a well-defined nucleus (see e.g. Fig. 2a), but four days later the comet reached magnitude +12.0 , exhibiting a completely different star-like appearance (Fig. 2b). The following days, the coma developed around the nucleus, and the released material was expanding outwards (Figs. 2d-g). On October 8 the comet exhibited a new outburst, and two days later the ejected material was observed to expand into the coma (Fig. 2h). The full sequence compiled in Fig. 2 is representative of the different appearances that SW1 exhibits depending of the time elapsed after an outburst.

Between July 2005 and March 2006, we imaged 6 outbursts typically of small amplitude where the SW1 coma increased its brightness by 1 or 2 mag (see e.g. Fig. 5). This observing period is shown in the detailed graph of Fig. 6 and shows the outbursts labeled $l$ to $q$. During this period the coma had the fan-shaped 


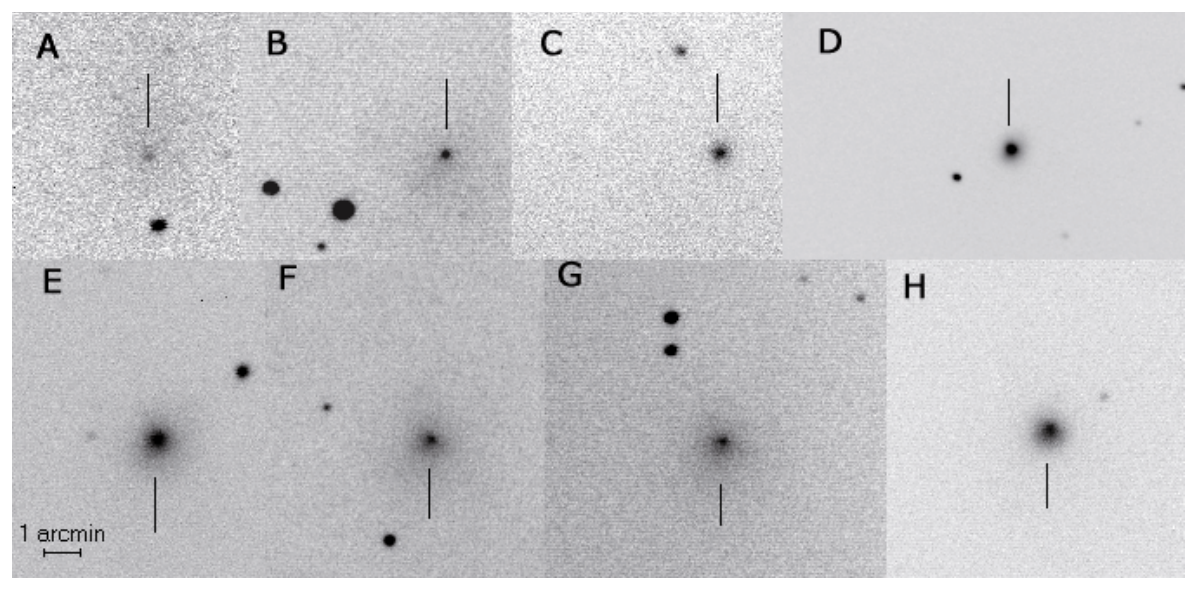

Fig. 2. The expanding coma of SW1 after an outburst detected on JD 2453266 (see magnitude profile in the outburst labeled as "i" in Fig. 6). All observations were obtained from Gualba Observatory (see telescope details in Table 1) with CCD Starlight Xpress MX716. Images taken on: a) JD 2453257,6 , b) JD 2453261,6 , c) JD 2453266,4, d) JD 2453267,7 , e) JD 2453273,6 , f) JD 2453280,4, g) JD 2453 282,3, h) JD 2453 289,4.

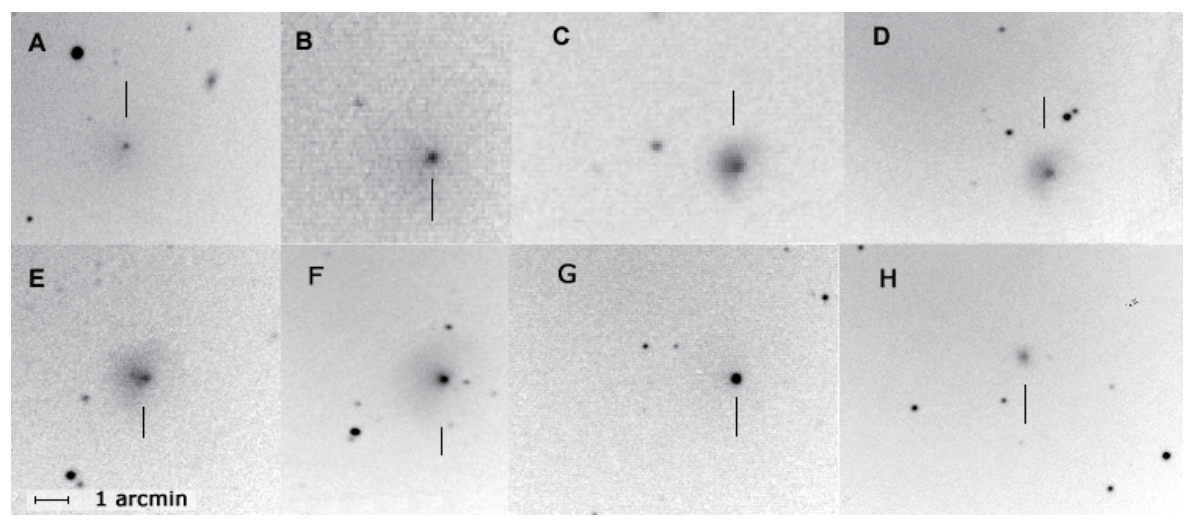

Fig. 3. The expanding coma of SW1 exhibiting a fan-shape after the outburst detected on JD 2453360 (see magnitude profile in the outburst labeled as "i" in Fig. 6). Identical instrumentation to Fig. 2. Images taken on: a) JD 2453350.2 , b) JD 2453353.3 , c) JD 2453359.3 , d) JD 2453364.3 , e) JD 2453368.3 , f) JD 2453373.3, g) JD 2453374.3 , h) JD 2453378.3.

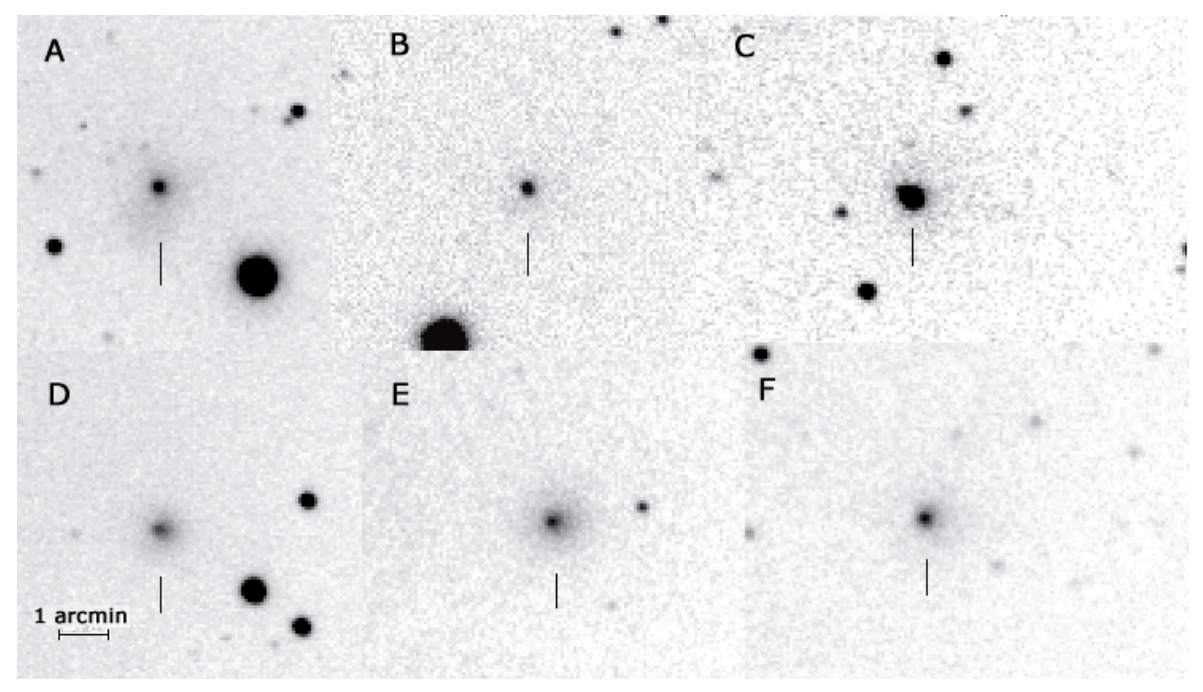

Fig. 4. Changes in the appearance of SW1. 2006 observations taken from Gualba Observatory (S/C 0,36 m $f=6$ ) with CCD CCD SBIG ST9 XE. Images taken on: a) JD 2453755.3 , b) JD 2453757.3 , c) JD 2453759.3 , d) JD 2453767.2 , e) JD 2453769.3 ,f) JD 2453774.3.

appearance that is produced by the anisotropic release of material from the surface. It is visible in Fig. 3, especially in Fig. 3d. In fact, the comparison of images would give clues to the nature of the decay process. The cloud of material is decreasing its luminosity with time as it expands. It is also important to point out that an outburst is followed by the development of an expanding coma as exemplified in Fig. 4. The outburst image (Fig. 4c) was taken on Janunary 23, 2006 (JD 2453759,25 ) when the comet increased its luminosity by 2 mag. This outburst was covered very well. The magnitude increased from +16.2 on Jan. 19 up to +14.4 on January 23,2006 . We also performed photometry in apertures of 20,30,40, and 60 arcsec. By using this procedure we were able to build the coma profile, to perform photometry for any aperture, and then to calculate the photometry for a constant physical aperture of any given diameter. From the coma diameters obtained by this approach we obtained Table 2 .

We performed a particularly successful coverage of the SW1 activity from July 2006 to December 2007. During this time interval, we identified eight different outbursts exhibiting very different amplitudes and with no clear periodicity (see the last graph in Fig. 6, just showing the outbursts labeled $r$ to $z$ ). The quiescent period of this comet between JD 2454070 and 2454127 (i.e. 57 days) is a rather typical time interval between outbursts. This widely extended period of apparent inactivity 

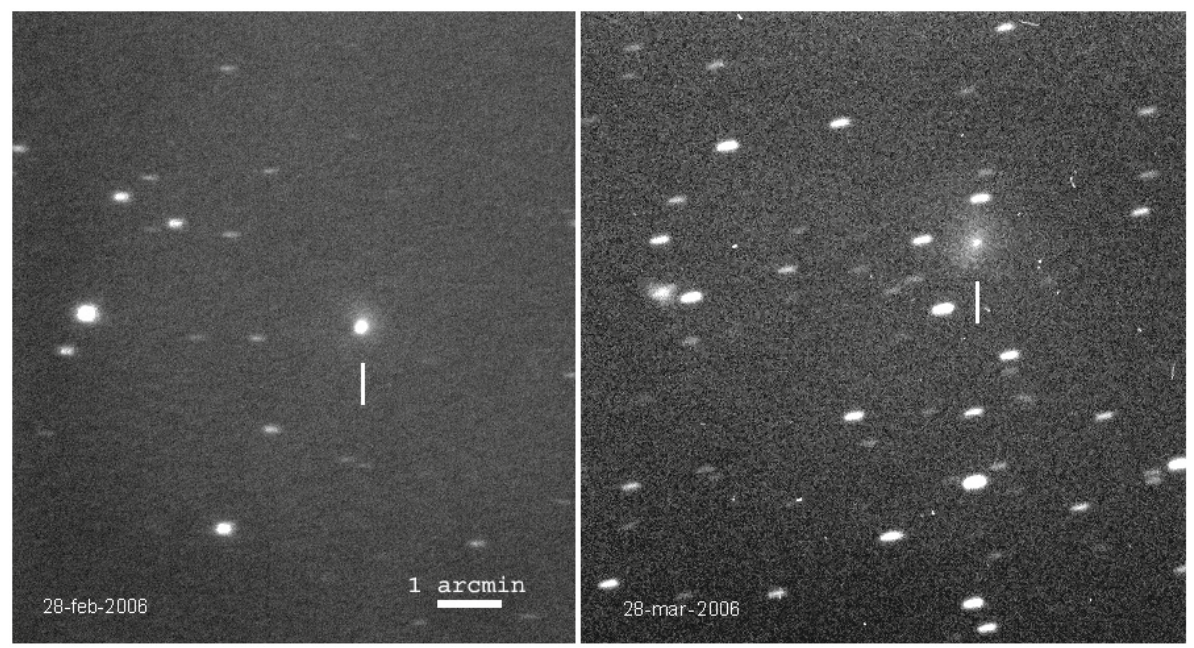

Fig. 5. The highly changing appearance of SW1 for medium-size telescopes. In the left image, the comet was suffering a small outburst (+14 mag) while the coma was widely developed in the right image. Images were taken from FOED by using a $61 \mathrm{~cm}$ Cassegrain telescope operated at primary focus $(6 \mathrm{~m})$ with a ST-9XE CCD.

Table 2. Selected measurements of the coma diameter of SW1. For more details see the text.

\begin{tabular}{llll}
\hline \hline Date (JD) & Image & $\begin{array}{l}\text { Apparent Coma } \\
\text { diameter }(\operatorname{arcsec})\end{array}$ & $\begin{array}{l}\text { Coma diameter } \\
\left(\times 10^{3} \mathrm{~km}\right)\end{array}$ \\
\hline 2453257.64 & Fig. 2a & 19.2 & $67 \pm 4$ \\
2453261.63 & Fig. 2b & 53.7 & $186 \pm 9$ \\
2453266.36 & Fig. 2c & 23 & $79 \pm 4$ \\
2453267.66 & Fig. 2d & 28.1 & $97 \pm 4$ \\
2453273.55 & Fig. 2e & 57.6 & $198 \pm 10$ \\
2453280.38 & Fig. 2f & 48.6 & $167 \pm 8$ \\
2453282.29 & Fig. 2g & 30.7 & $105 \pm 5$ \\
2453289.36 & Fig. 2h & 33.2 & $115 \pm 6$ \\
\hline
\end{tabular}

ended with a 3-mag outburst around JD 2454 130. After the decay in brightness, two additional outbursts occurred, increasing the overall activity of the comet.

To estimate the number of outbursts per year, we compiled the observational data statistics in Table 3 . The data sample involves photometric measurements for 1400 days where 28 outbursts were clearly identified. Of course, this number is a lower limit because our coverage is typically about $30 \%$ of the nights. In any case, due to the quite homogenously distributed sampling and the extent of the outburst period for more than a week, we probably missed only few outbursts. Taking all this into account, we obtain about 7.3 outbursts/year, which is higher than the 1-2 outbursts/year estimated earlier (see e.g. Cook et al. 2005).

By analyzing all available data, we did not find a clear dependence of the outburst activity as a function of the distance to the Sun as is shown in Fig. 7. Possible periodicities in the outburst activity of this comet were also studied for the full sample and for selected time periods. Because the observational intervals are unevenly spaced, we used different tools in the periodogram analysis. Basically we used the phase dispersion minimization (PDM) (Stellingwerf 1978) and the discrete Fourier transform (Deeming 1975) methods. For simplicity we only include the PDM periodograms. The resulting periodograms are shown in Fig. 8. There is a clear peak and aliases (i.e. period combinations) for the synodic period of about 392 days, and another quite evident period of 1 cycles/day as a consequence of the typical 24-h observational window. However, we also found two possible peaks at frequencies of 0.001 cycles/day and another at 0.05 cycles/day (Period $=50$ days), which are not easily explained. However, since the signal-to-noise ratios of about 3.5 are below the 4.5 level required for a solid detection, both periodicities remain uncertain.

\section{Discussion}

\subsection{The origin and nature of SW1 outbursts}

The solar radiation absorbed at a comet nucleus surface is dissipated by thermal reradiation into space, sublimation of surface ice, and conduction into the interior as described by the energy balance equation,

$$
\frac{S_{\odot}(1-A) \cos \zeta(t)}{r_{\mathrm{h}}^{2}}=\varepsilon \sigma T^{4}+L \cdot p_{\text {sat }} \sqrt{\frac{m_{\mathrm{H}_{2} \mathrm{O}}}{2 \pi k T}}-\kappa \frac{\mathrm{d} T}{\mathrm{~d} x}
$$

where $S_{\odot}=1367 \mathrm{~J} \mathrm{~m}^{-2} \mathrm{~s}^{-1}$ is the solar constant, $A=0.04 \mathrm{a}$ representative Bond albedo, $\zeta(t)$ the time-dependent solar zenith angle, $\varepsilon=1$ a representative emissivity, $\sigma$ the Stefan-Boltzmann constant, $p_{\text {sat }}(T)=3.56 \times 10^{12} \exp (-6141.667 / T)[\mathrm{Pa}]($ Fanale $\&$ Salvail 1984) the saturation pressure of water vapor, $m_{\mathrm{H}_{2} \mathrm{O}}$ the water molecule mass, $k$ the Boltzmann constant, $L=2.6 \times$ $10^{6} \mathrm{~J} / \mathrm{kg}$ the latent heat, and $\kappa$ the conductivity. Evaluating this equation for a heliocentric distance of $6 \mathrm{AU}$ within the limit of negligible heat conduction, an upper limit on the noon temperature of $T \approx 160 \mathrm{~K}$ is obtained. At such a low temperature, $99.5 \%$ of the absorbed energy is reradiated thermally, and the water sublimation rate is $8.7 \times 10^{-8} \mathrm{~kg} \mathrm{~m}^{-2} \mathrm{~s}^{-1}$. This is completely negligible compared to e.g. the peak sublimation rate of $4.6 \times 10^{-4} \mathrm{~kg} \mathrm{~m}^{-2} \mathrm{~s}^{-1}$, expected at $r_{\mathrm{h}}=1 \mathrm{AU}$. For the secondary species $\mathrm{CO}$ and $\mathrm{CO}_{2}$, the same high sublimation rate of $4.6 \times$ $10^{-4} \mathrm{~kg} \mathrm{~m}^{-2} \mathrm{~s}^{-1}$ would take place at temperatures as low as $T=38 \mathrm{~K}$ and $T=113 \mathrm{~K}$, respectively, using saturation pressure formulae from Tancredi et al. (1994) and Orosei et al. (1995). For comet SW1, one would therefore expect that a surface layer (perhaps ten meters in thickness) should be completely void of pure $\mathrm{CO}$ and $\mathrm{CO}_{2}$ condensates. But if no highly volatile species are present in a pure form near the surface and if water ice is far too cold to sublimate, what is causing the outbursts?

Several outburst mechanisms have been proposed, including polymerization of HCN (Rettig et al. 1992) and meteoroid impacts (Gronkowski 2004). However, the perhaps most reasonable explanation put forward to date is crystallization of amorphous water ice (Smoluchowski 1981; Prialnik \& Bar-Nun 1987). Amorphous ice crystallizes irreversibly and exothermally around $137 \mathrm{~K}$ and the released energy could be the source driving the outburst, as well as being responsible for release of $\mathrm{CO}$ 

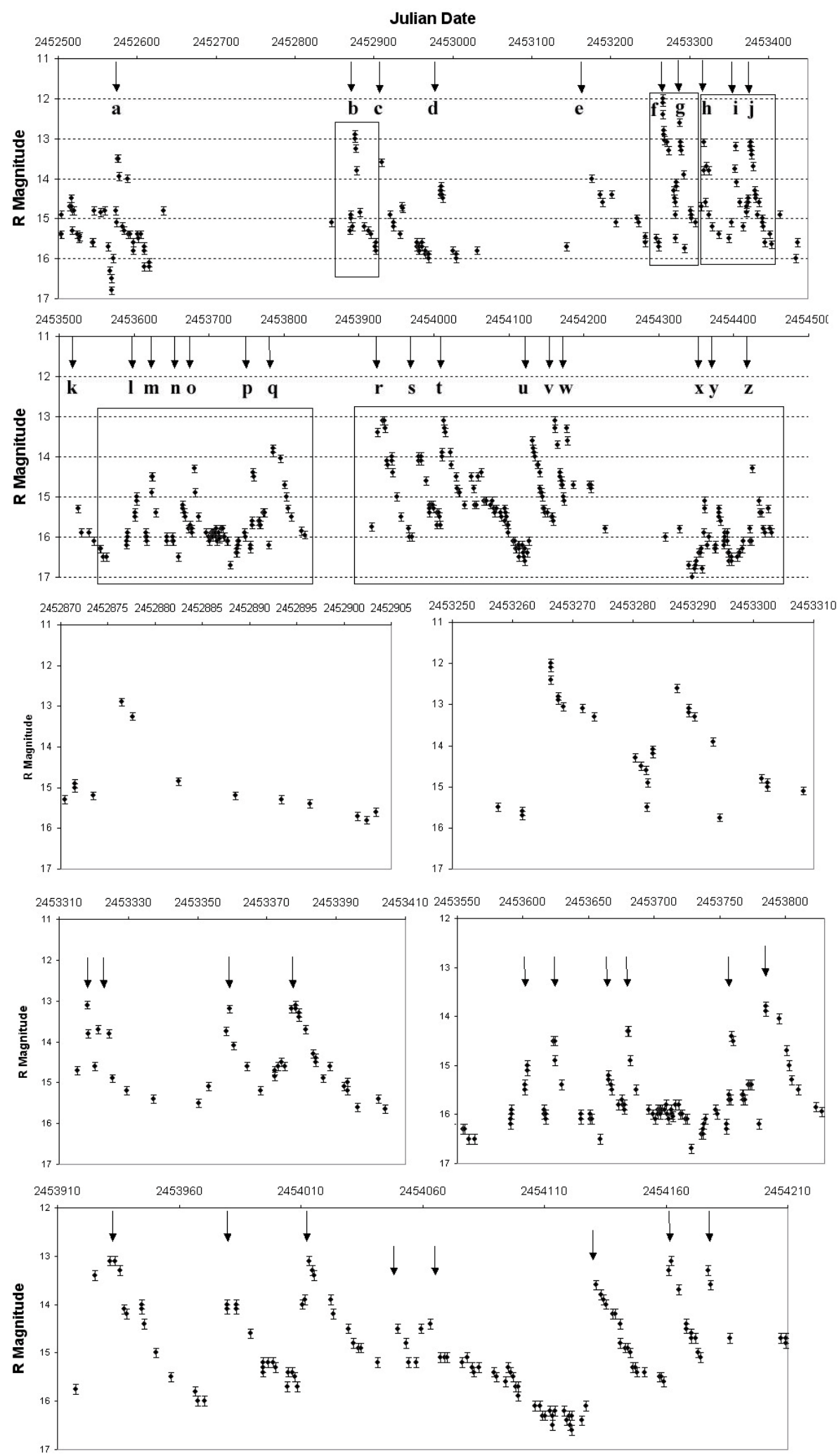

Fig. 6. Two extended graphs with the 10 arcsec photometry in the filter $R$ for comet SW1 according to all our data. Five windows showing selected outbursts are shown below in detail. At that size the error bars become visible. The $3 \sigma$ errors are shown in the data points.

and $\mathrm{CO}_{2}$ being trapped in the water matrix. This has been described and modeled for comet C/1995 O1 Hale-Bopp (Prialnik 1997). In this work the increase in CO emission was correlated with the runaway transition of amorphous to crystalline ice that contributes to the release of gases initially trapped in the ice. At the same time, this process could perhaps explain the curious effect observed by Cochran et al. (1982) consisting in the $\mathrm{CO}^{+}$emission turning on and off on a very rapid timescale.
The energy release by crystallization will produce a local subsurface temperature maximum, causing heat to flow both towards the surface and towards the deeper interior. As a consequence of these processes, the progressive release of occluded gas and fine dust takes place flowing through the pores (Prialnik 2002). The transition of amorphous to crystalline ice is an exothermic process contributing to the weakening of the superficial structure and to increase the empty volume occupied by 
Table 3. Statistics of the data presented in Fig. 6, making special emphasis in the number of detected outburst during each observing campaign.

\begin{tabular}{lllll}
\hline \hline $\begin{array}{l}\text { Observing period } \\
\text { (JD) }\end{array}$ & $\begin{array}{l}\text { Observing } \\
\text { period (yrs) }\end{array}$ & $\begin{array}{l}\text { Observed } \\
\text { nights }\end{array}$ & $\begin{array}{l}\text { Coverage } \\
\text { (\% of nights) }\end{array}$ & $\begin{array}{l}\text { Number of detected } \\
\text { outbursts* }\end{array}$ \\
\hline $2452500-2452633$ & Aug.-Dec. 2002 & 38 & 27.5 & 3 \\
$2452845-2453032$ & Jul. 03-Jan. 04 & 37 & 17.4 & 3 \\
$2453144-2453438$ & May 04-Mar. 05 & 65 & 22.2 & 6 \\
$2453525-2453828$ & Jun. 05-Abr. 06 & 81 & 26.7 & 7 \\
$2453917-2454228$ & Jul. 06-May 07 & 105 & 33.8 & 6 \\
$2454309-2454450$ & Jul. 07-Dec. 07 & 39 & 27.8 & 3 \\
\hline
\end{tabular}

* An outburst is counted when an increase of at least 1 mag in the brightness of the comet is noticed.

pores. As the flow of gases and small dust occurs, this process introduces changes in the strength of the cometary surface. In fact, it has been suggested that the fracture and ejection of relatively large areas in SW1 surface could be producing large noncircular depressions in the nucleus like those observed in comet Wild 2 (Brownlee et al. 2004). Prialnik \& Bar-Nun (1992) propose major explosions produced by the exposition to solar radiation when the superficial layer breaks apart. Another possibility suggested by Laufer et al. (2005) is the chaotic collapse of the dust-covered crystalline ice layer, which also exposes pristine ice to solar heating. Laboratory simulations made by Laufer et al. (2005) produced submillimeter-to-centimeter ice particles that can be released from active regions during chaotic collapse of an outer ice layer. Then, the collapse and ulterior ejection of ice layers overlying gas pockets is our suggested mechanism for renewal of cometary activity. This type of activity could perhaps explain the non-circular active depressions observed in the surfaces of several comets (Brownlee et al. 2004).

\subsection{Dust behavior in the SW1 coma}

Our observations show that the SW1 coma is continuously supplied with fine dust. Even when the object is really faint (mag +16$)$, it keeps its diffuse appearance, and the coma extends about $10^{5} \mathrm{~km}$ from the nucleus. Such a continuous release of dust is also supported by Spitzer observations that detected a jet even when the comet was not in outburst (Stansberry et al. 2004). These are important observations, since it indicates that a low-level activity is also maintained between outbursts.

According to our data, a typical outburst shows a progressive decay in brightness to a nearly initial state in about 3-4 days. If this decay is solely due to the dilution caused by expansion, and if we take $10^{5} \mathrm{~km}$ as the limit beyond which dust is difficult to detect, it would imply an average dust velocity on the order of $0.3 \mathrm{~km} \mathrm{~s}^{-1}$. This is in fact somewhat high compared to the expansion velocity of $0.15 \mathrm{~km} \mathrm{~s}^{-1}$ observed during an SW1 outburst (Feldman et al. 1996), which perhaps could indicate that an additional mechanism for brightness decrease is active. In this context it is interesting to note that the 3-4 day time-frame coincides with the expected lifetime of the optically effective icy grains, the reason being the following. Observations of comet dust grains in the visual only samples a limited part of the grain size distribution centered around $1 \mu \mathrm{m}$. Smaller grains are invisible due to a rapid decrease of the scattering coefficient with diminishing size (Rayleigh limit), while larger grains do not contribute substantially to the observed light due to their small number. Hence, what is seen in our observations are primarily $\mu \mathrm{m}$-sized grains. According to Gunnarsson (2003), who performed grain lifetime calculations for clusters of sub- $\mu \mathrm{m}$ coremantle particles, the lifetime of $\mu \mathrm{m}$-sized clusters is indeed a few times $10^{5} \mathrm{~s}$, or a couple of days. Although it is premature to suggest that grain sublimation and fragmentation are partially responsible for the observed brightness decay without proper modeling, we certainly consider it an interesting topic for future studies.

Gunnarsson et al. (2002) also finds a extended CO source in the coma, which was later interpreted as evidence of sublimating icy grains (Gunnarsson 2003). According to these authors, the CO-producing material is located outside the nucleus at a distance of $1-2 \times 10^{5} \mathrm{~km}$, having an estimated travel time of order $2-4 \times 10^{6} \mathrm{~s}$. This would give the responsible grains an approximate velocity of $50 \mathrm{~m} \mathrm{~s}^{-1}$, which is substantially lower than the values quoted above. This probably reflects the reasonably lower dust velocities between outbursts (their observations were made during a longer "quiet" period), as well as a lower velocity expected for heavier particles since they are less accelerated by gas drag (the lifetime, equal to the travel time, suggest particle sizes of a few microns). We find it interesting that the distance at which the extended CO-source is located coincides with the typical size of the visible coma in our observations, which perhaps could indicate that a rapid disintegration of grains is observed. However, we wish to repeat that modeling the coma brightness profile is needed before concluding that dilution alone is insufficient for explaining the observations.

\section{Conclusions}

Remote observations of comet SW1 by using medium-sized telescopes provide valuable information on the mechanisms capable of explaining its typical outburst behavior. We have shown that a monitoring of the activity of SW1 can provide clues to the origin of cometary outbursts. Continuity in the observations is important because incomplete statistics will hamper any search for periodicity in the outburst production. In any case, we think that a spacecraft mission to this fascinating comet would give additional clues to the physical mechanisms involved in cometary activity. To summarize, we found:

1. Photometry of cometary comae using the 10 arcsec standardized method is giving valuable information on the fluctuation of the coma brightness. Observations made by different instruments are giving similar values up to (at least) a tenth of a magnitude.

2. Typical SW1 outbursts occur with a frequency of 7.3 outbursts/year and are characterized at the present time by the sudden increase of 1 to 4 mag in the brightness of SW1. The PDM periodograms show no clear periodicity in the outburst production. Highly changing surface processes are suspected to be responsible for the observed behavior.

3. Fracture of weak surface regions, their ulterior fragmentation, and ejection by subsurface explosions would impulse less than one-mm-sized particles into the coma. As $100 \mu \mathrm{m}$ particles are virtually unobservable in cometary comae at 


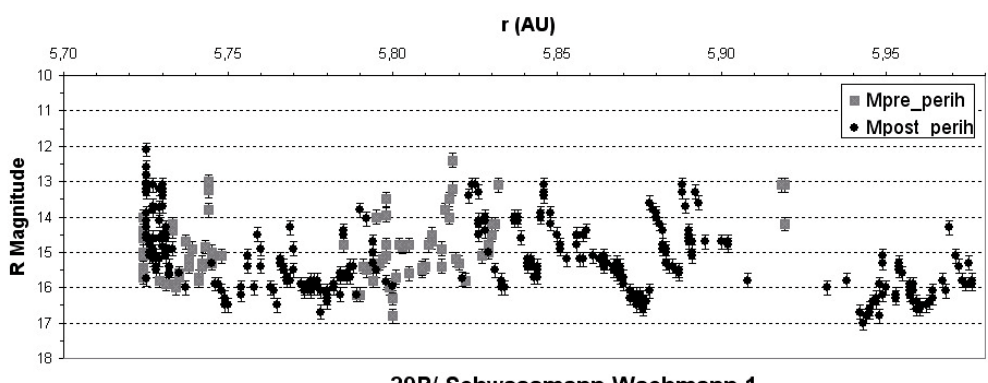

29P/ Schwassmann-Wachmann 1
Fig. 7. Comet magnitude as a function of the heliocentric distance. Pre-perihelion (Mpre_perih) and post-perihelion (Mpost_perih) magnitudes are compared. Note that the amplitude of SW1 outburst activity does not show any correlation with heliocentric distance. The $3 \sigma$ errors are shown in the data points.
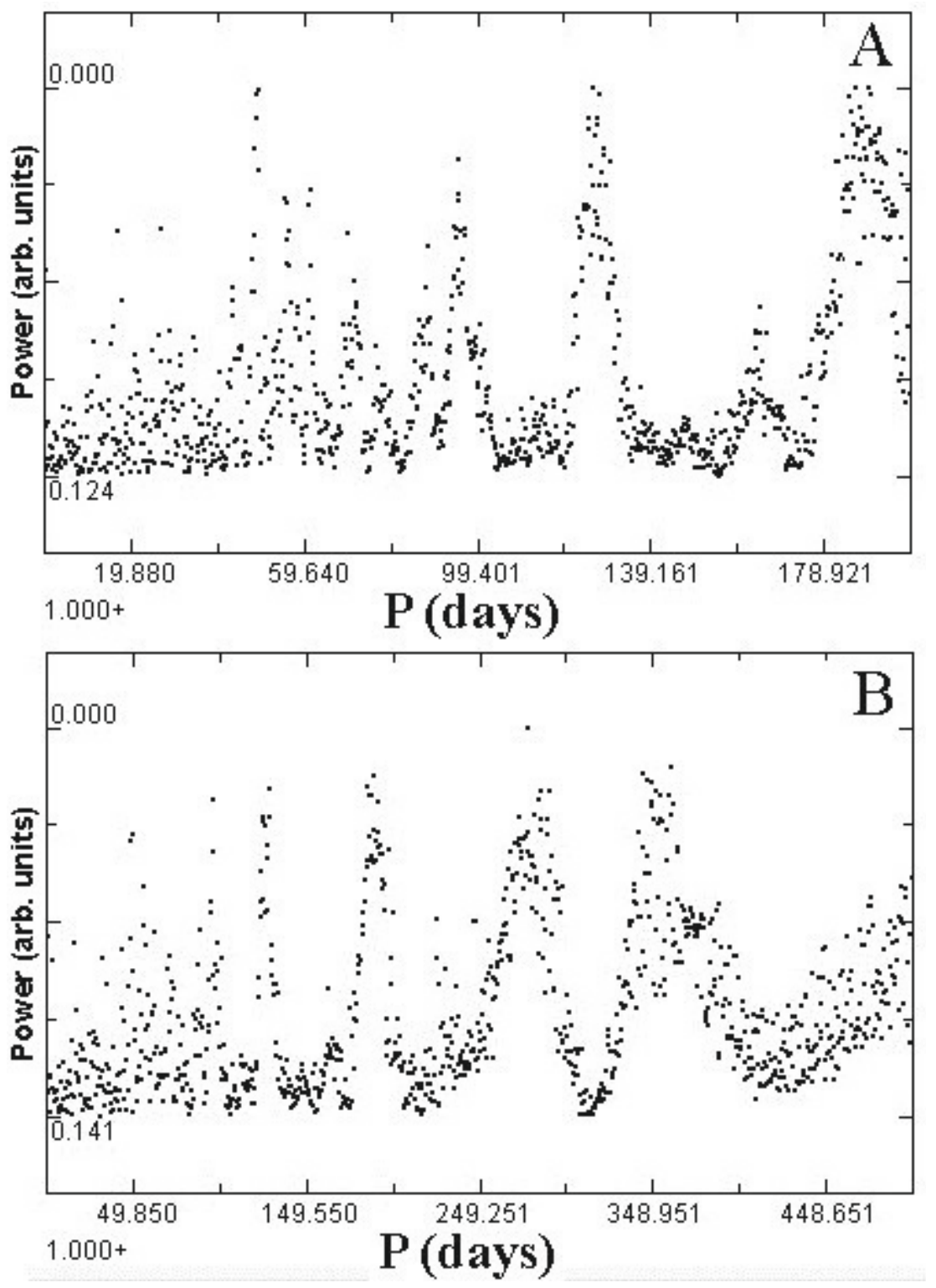

Fig. 8. Phase dispersion minimization (PDM) periodograms for two different period windows in order to see the different peaks and aliases discussed in the text.

visual wavelengths, subsequent fragmentation into smaller pieces would reflect more light, creating the sudden magnitude increase.

4. Fragmentation of the released (likely ice-rich) particles because of sublimation by solar heating could explain the observed diameter, development, and decay of the SW1 coma after an outburst.

5. The decay in brightness of the coma after an outburst is expected from the expansion of the grains, due to a decrease in the spatial number density of dust grains. It is also possible that the progressive sublimation of ice-rich grains (a subsequent darkening of remaining $\mathrm{C}$-rich particles) would produce a steeper decrease in the overall coma luminosity. This topic should be studied in the future by performing calibrated measurements of the coma evolution using narrowband filters.

6. Finally, we think that a continuous monitoring of comets by using medium-size telescopes and CCD cameras under a systematic procedure is valuable for completing professional programs requiring wide coverage. 
Acknowledgements. J.M.T.R. thanks Ministerio de Educación y Ciencia (MEC) for a JdC grant. We also thank Dr. David Fernández (IEEC) for technical support with the Joan Oró $0.8 \mathrm{~m}$ telescope of the Montsec Astronomical Observatory (OAdM). The authors thank Grup d'Estudis Astronòmics (GEA) for providing the reduction software used in the present studies, especially LAIA and AVE programs developed by R. Barberà and J. A. Cano.

\section{References}

Brownlee, D. E., Anderson, J. D., Atkins, K., et al. 2004, Lunar Planet. Sci., 35, Abst. \#1981

Cochran, A. L., Cochran, W. D., \& Barker, E. S. 1982, ApJ, 254, 816

Cook, J. C., Desch, S. J., \& Wyckoff, S. 2005, BAAS, 37, 645

Deeming, T. J. 1975, Astrophys. Space Sci., 36, 137

Escolà-Sirisi, E., Juan-Samsó, J., Vidal-Sáinz, J., et al. 2005, A\&A, 434, 1063

Fanale, F. P., \& Salvail, J. R. 1984, Icarus, 60, 476

Feldman, P. D., McPhate, J. B., Weaver, H. A., Tozzi, G.-P., \& A'Hearn, M. F. 1996, BAAS, 28, 1084

Froeschlé, Cl., Linger, J., \& Rickman, H. 1983, in Asteroids, Comets, Meteors, A85-26851 11-89, 215

García-Melendo, E., \& Clement, C. M. 1997, AJ, 114, 1190

Green, D. W. E., et al. 2000, IAU Circular, \#7605

Green, D. W. E., et al. 2007, IAU Circular, \#8886

Gronkowski, P. 2004, Astron. Nachr., 325, 343

Gunnarsson, M. 2003, A\&A, 398, 353

Gunnarsson, M., Rickman, H., Festou, M. C., Winnberg, A., \& Tancredi, G. 2002, Icarus, 157, 309
Kidger, M. 2002, EMP, 90, 259

Kidger, M. 2003, A\&A, 408, 767

Laufer, D., Pat-El, I., \& Bar-Nun, A. 2005, Icarus, 178, 248

Marsden, B., \& Williams, G. V. 2003, Catalogue of cometary orbits, 15th Ed., USA

Orosei, R., Capaccioni, F., Capria, M. T., et al. 1995, A\&A, 301, 613

Prialnik, D. 1997, ApJ, 478, L107

Prialnik, D. 2002, EM\&P, 89, 27

Prialnik, D., \& Bar-Nun, A. 1987, ApJ, 313, 893

Prialnik, D., \& Bar-Nun, A. 1990, ApJ, 363, 274

Prialnik, D., \& Bar-Nun, A. 1992, A\&A, 258, L9

Raab, H. 2005, Astrometrica software, Shareware for research grade CCD Astrometry: http: //www . astrometrica.at/

Rettig, T. W., Tegler, S. C., Pasto, D. J., \& Mumma, M. J. 1992, ApJ, 398, 293

Senay, M. C., \& Jewitt, D. 1994, Nature, 371, 229

Sekanina, Z. 1982, in Comets, ed. L. L. Wilkening (Tucson: Univ. of Arizona), 251

Sekanina, Z., Boehnhardt, H., Käufl, H. U., \& Birkle, K. 1996, in JPL Cometary Sciences Group Preprint Series, 183, 1

Smoluchowski, R. 1981, ApJ, 244, L31

Stansberry, J. A., Van Cleve, J., Reach, W. T., et al. 2004, ApJS, 154, 463

Stellingwerf, R. F. 1978, ApJ, 224, 953

Tancredi, G., Rickman, H., \& Greenberg, J. M. 1994, A\&A, 286, 659

Trigo-Rodríguez, J. M., et al. 2007, Comet 17P/Holmes, CBET 1118, IAU

Trigo-Rodríguez, J. M., Davidsson, B., Montañés-Rodríguez, P., Sánchez, A., \& Troughton, B. 2008, Lunar Planet. Sci.39, abstract \#1627 (Houston: Lunar and Planetary Institute)

Wyckoff, S. 1982, in Comets, ed. L. L. Wilkening (Tucson: Univ. of Arizona), 3 